\title{
Effects of Guar Gum and Fructooligosaccharides on Plasma Lipids and Cecal Short-Chain Fatty Acids in Adult Mice
}

\author{
Motoi Tamura, ${ }^{1, *}$ Hiramitsu Suzuki, ${ }^{1}$ Kazuhiro Hirayama, ${ }^{2}$ and Kikuji ItoH $^{2}$ \\ ${ }^{1}$ National Food Research Institute, Ministry of Agriculture, Forestry and Fisheries, \\ Tsukuba 305, Japan \\ ${ }^{2}$ Department of Veterinary Public Health, Faculty of Agriculture, \\ The University of Tokyo, Bunkyo-ku, Tokyo 113, Japan
}

(Received June 23, 1997)

\begin{abstract}
Summary The effects of fructooligosaccharides added to guar gum on plasma lipids and cecal short-chain fatty acids (SCFAs) in mice were studied. Male Crj:CD-1 (ICR) mice were fed an MF diet for 34 weeks, and then the MF diet was replaced with a semisynthetic diet supplemented with $5 \%$ guar gum or $5 \%$ guar gum plus $3 \%$ fructooligosaccharides. The mice fed the guar gum or guar gum-fructooligosaccharide diet for 3 weeks. There was no significant difference in cecal bacterial counts or cecal content between the two diet groups. There was, however, a significant difference in their plasma triglyceride concentrations. The plasma triglyceride concentration was significantly lower in the mice fed the guar gum diet than in those fed the guar gum-fructooligosaccharide diet. However, no significant difference in plasma total cholesterol concentration was noted between the two diet groups. The butyric acid concentration in the guar gum group was significantly higher than that in the guar gum-fructooligosaccharide group. No significant difference in propionic acid or acetic acid concentration was noted between the groups. The difference in the plasma triglyceride concentration between the guar gum and guar gum plus fructooligosaccharide groups might be related to a difference in floral metabolism, floral composition, or both.
\end{abstract}

Key Words: guar gum, fructooligosaccharides, plasma lipids, SCFAs

The role of dietary fiber in nutrition is now widely recognized, including a plasma cholesterol-lowering effect [1-3] and a modification of intestinal absorption of dietary lipids [4]. Various types of gel-forming polysaccharides, such as gums, may display different effects on transit time, or on the excretion of fecal fat

* To whom correspondence should be addressed. 
and fecal bile acids $[5,6]$. Like dietary fiber, fructooligosaccharides escape digestion in the small intestine and enter the cecum without significant changes in their structure. Both guar gum and fructooligosaccharides are fermented by the resident microflora [7-9]. Short-chain fatty acids (SCFAs) are the main products formed during fermentation of carbohydrates in the large bowel. A large proportion of the metabolic effects of dietary fiber could be due to its degradation to SCFAs, which have considerable effects on both intestinal and liver metabolism. However, there is little information of the composite effect of dietary fiber on plasma lipid and cecal SCFAs. The aim of the present study was to examine the effects of fructooligosaccharides added to guar gum on plasma lipids and cecal SCFAs.

\section{MATERIALS AND METHODS}

Animals and diets. Corn oil was supplied by NOF Corp., Tokyo. Guar gum was obtained from Sigma Chemical Co., St. Louis, MO, and was intact. Fructooligosaccharides were obtained from Wako Pure Chemical Industries, Ltd., Osaka. Fructooligosaccharides comprised 43.4\% (w/w) 1-kestose, $45.8 \%(\mathrm{w} / \mathrm{w})$ nystose, 7.3\% (w/w) 1-fructofuranosyl-D-nystose. Male Crj:CD-1 (ICR) mice (5 weeks old) obtained from Charles River Japan (Atsugi, Kanagawa) were randomly divided into three groups of seven animals each. One group was used as the initial group. The animals were housed individually in suspended stainless-steel cages with wire mesh bottoms. The room was kept at $24 \pm 0.5^{\circ} \mathrm{C}$ and a relative humidity of $65 \%$. Room lighting consisted of alternating 12 -h periods of light and dark. The animals were fed the MF diet (Oriental Yeast Co., Ltd., Tokyo) for 34 weeks. The mice in the initial group were anesthetized with diethylether, and cecal SCFAs and plasma lipids in samples obtained from them were measured. In the other two groups, the MF diet was replaced with the guar gum diet (group 1) or the guar gumfructooligosaccharide diet (group 2). Both diet and water were provided ad libitum for 3 weeks. The guar gum diet contained 5\% guar gum and $10 \%$ sucrose, the guar gum-fructooligosaccharide diet contained 5\% guar gum, 3\% fructooligosaccharides, and $7 \%$ sucrose, and the remaining dietary components in both groups were corn starch, $48.8 \%$; casein, $25.0 \%$; corn oil, $5 \%$; mineral mixture, $4.0 \%$; vitamin mixture, 2.0\%; and L-methionine, $0.2 \%$. Mineral and vitamin mixtures were purchased from Oriental Yeast Co., Ltd. Their composition was described by Kohashi et al. [10]. Body weight gain and food consumption were measured.

Sampling. At the end of the feeding trial, all mice were anesthetized with diethylether, and blood was collected from the inferior vena cava. The blood was centrifuged, and the plasma was frozen below $-20^{\circ} \mathrm{C}$ until analyzed. Cecal contents were collected and processed immediately for measurement of bacterial counts and cecal SCFAs.

Bacteriological procedures. Cecal samples were weighed in both groups 1 and 2. Serial 10-fold dilutions of the samples were spread on the surface of EG 
agar media, and then incubated for 3 days at $37^{\circ} \mathrm{C}$ in a Gas Pak anaerobic system (Becton Dickinson and Company, Cockeysville) filled with hydrogen and carbon dioxide. Bacterial numbers were expressed as $\log _{10}$ counts of viable bacteria per gram wet of cecal content.

Measurement of cecal SCFAs concentration. Measurement of cecal SCFAs concentration was essentially the same as described by Hirayama et al. [11] except that a capillary column was employed instead of a glass column. Cecal samples were acidified with $50 \% \mathrm{H}_{2} \mathrm{SO}_{4}$. Crotonic acid was added as an internal standard, and diethyl ether was used to extract the SCFAs. The ether extract was injected into a gas chromatograph equipped with a flame ionization detector (GC-14A, Shimadzu Seisakusho Ltd., Kyoto) and a capillary column $(30 \mathrm{~m} \times 0.25 \mathrm{~mm}$, TC-FFAP, GL Sciences Ltd., Tokyo). Other operating parameters were as follows: carrier gas of $\mathrm{N}_{2}, 1.25 \mathrm{~kg} / \mathrm{cm}^{2}$; temperature of column oven, $150^{\circ} \mathrm{C}$; temperature of injection port and detector, $250^{\circ} \mathrm{C}$.

Measurement of plasma lipids. Total plasma cholesterol, and triglyceride concentrations were determined by the methods of Allain et al. [12], Spayd et al. [13], respectively.

Statistical analysis. All results are reported as the mean \pm SD. Student's $t$ test was used to determine statistical significance of the differences between mean values.

\section{RESULTS}

There was no significant difference in food consumption (g/day) or body weight between the guar gum and guar gum-fructooligosaccharide diet groups.

\section{Cecal bacterial count and cecal content}

There was no significant difference in cecal bacterial counts or weight of cecal contents between the two diet groups (Table 1).

\section{Plasma lipid concentration}

The plasma lipid concentrations of the mice fed the guar gum diet, guar gum-fructooligosaccharide diet, and MF diet are shown in Table 2. There was a significant difference in the plasma triglyceride concentration between the animals

Table 1. Cecal content and cecal bacteria count of the mice fed the two different diets.

\begin{tabular}{lrr}
\hline & \multicolumn{2}{c}{ Dietary groups } \\
\cline { 2 - 3 } & Guar gum & Guar gum + fructooligosaccharides \\
\hline Cecal content (Wet weight: g) & $0.15 \pm 0.07$ & $0.18 \pm 0.06$ \\
Cecal bacterial count ${ }^{\mathrm{a}}$ & $10.62 \pm 0.40$ & $10.60 \pm 0.42$ \\
\hline \multicolumn{2}{l}{ Values are means \pm SD $(n=7){ }^{\mathrm{a}}$ Mean \pm SD of $\log _{10}$ count of viable bacteria per gram cecal } \\
content.
\end{tabular}

Vol. 23, No. 2, 1997 
Table 2. Plasma lipid concentrations of the mice fed the three different diets.

\begin{tabular}{lccc}
\hline & \multicolumn{3}{c}{ Dietary groups } \\
\cline { 2 - 4 } & MF (Initial group) & Guar gum & Guar gum + fructooligosaccharides \\
\hline Cholesterol $(\mathrm{mg} / \mathrm{dl})$ & $144.0 \pm 34.0$ & $134.9 \pm 30.1$ & $152.4 \pm 22.3$ \\
Triglyceride $(\mathrm{mg} / \mathrm{dl})$ & $95.2 \pm 37.6^{*}$ & $53.2 \pm 11.8$ & $80.2 \pm 11.3^{* *}$ \\
\hline
\end{tabular}

Values are means \pm SD $(n=7)$. ${ }^{*}$ Significantly different $(p<0.05)$ from guar gum diet group.

**Significantly different $(p<0.01)$ from guar gum diet group.

Table 3. Concentrations of SCFAs in the cecum of the mice fed the three different diets $(\mu \mathrm{mol} / \mathrm{g})$.

\begin{tabular}{lccc}
\hline & \multicolumn{3}{c}{ Dietary groups } \\
\cline { 2 - 4 } & MF (Initial group) & Guar gum & Guar gum + fructooligosaccharides \\
\hline Total SCFAs & $52.9 \pm 19.1$ & $51.2 \pm 15.0$ & $40.3 \pm 13.0$ \\
Acetic acid & $36.4 \pm 13.9$ & $33.9 \pm 10.5$ & $26.9 \pm 9.3$ \\
Propionic acid & $8.7 \pm 3.3$ & $8.0 \pm 2.7$ & $7.1 \pm 2.8$ \\
$i$-Butyric acid & $0.9 \pm 0.3$ & $0.7 \pm 0.2$ & $0.7 \pm 0.4$ \\
Butyric acid & $4.8 \pm 1.5$ & $7.0 \pm 2.5$ & $4.0 \pm 1.2^{*}$ \\
$i$-Valeric acid & $1.1 \pm 0.3$ & $0.8 \pm 0.2$ & $1.0 \pm 0.4$ \\
Valeric acid & $0.9 \pm 0.2$ & $0.9 \pm 0.4$ & $0.8 \pm 0.3$ \\
\hline
\end{tabular}

Values are means \pm SD $(n=7)$. ${ }^{*}$ Significantly different $(p<0.05)$ from guar gum diet group.

fed the guar gum and the guar gum-fructooligosaccharide diets, and between those fed the guar gum and the MF diet (Table 2). The plasma triglyceride concentration was significantly lower in the mice fed the guar gum diet than in those fed the guar gum-fructooligosaccharide diet. However, no significant difference in plasma total cholesterol concentration was noted between these two groups (Table 2).

\section{Cecal SCFAs}

Cecal SCFAs concentrations in the mice fed the guar gum diet, guar gumfructooligosaccharide diet, and MF diet are shown in Table 3. The butyric acid concentration in the guar gum diet group was significantly higher than that in the guar gum-fructooligosaccharide diet group (Table 3). However, there was no significant difference in the concentrations of propionic acid and acetic acid between the two groups (Table 3 ). The ratio of propionic acid to butyric acid in the cecum of the mice fed guar gum diet $(1.2 \pm 0.4)$ was significantly lower than that in those fed the guar gum-fructooligosaccharide diet $(1.8 \pm 0.4)$ or MF diet $(1.8 \pm 0.4)$. However, there were no significant differences in the ratio of propionic acid to acetic acid or acetic acid to butyric acid between the mice fed the guar gum and guar gum-fructooligosaccharide diets. 


\section{DISCUSSION}

There was no significant difference in the bacterial cecal population between the mice fed guar gum and those fed guar gum-fructooligosaccharides. It is reported that guar gum and fructooligosaccharides are almost completely utilized by the hind-gut microflora, about $90-100 \%$ being fermented [9]. Thus, both guar gum and fructooligosaccharides should be well fermented in the cecum of mice. Fructooligosaccharides are fermented by only a limited range of microorganisms, including Bacteroides and all species of bifidobacteria except B. bifidum [14]. The ratio of bifidobacteria to total bacteria has been shown to increase with administration of a $0.1 \%$ fructooligosaccharide solution for 4 months in human floraassociated mice [15]. Guar gum is also completely utilized by the hind-gut microflora [9]. The floral composition in mice fed guar gum plus fructooligosaccharides might therefore be different from that of mice fed guar gum. It is reported that fecal in vitro butyrate production is greater for guar gum-fed rats than for control rats after 3-11 weeks of diet treatment [16]. Furthermore, it is reported that oligofructose- and fructooligosaccharide-containing diets result in a higher cecal butyrate concentration compared with control, cellulose diets [17]. From these reports, both guar gum and fructooligosaccharide diets might be expected to result in a higher butyrate concentration in the cecum compared with the control diet. However, we found that the cecal butyrate concentration in mice fed the guar gum-fructooligosaccharide diet was significantly lower than that in mice fed the guar gum diet. The floral composition or floral metabolism of mice fed guar gum might be different from that of mice fed the guar gum-fructooligosaccharide one. The addition of fructooligosaccharides to guar gum might alter the floral composition or floral metabolism from the situation prevailing when mice are fed guar gum alone. It is also reported that the serum triglyceride-lowering effect of oligofructose, which is chemically similar to that of fructooligosaccharides, may be related to SCFAs and/or lactate, which are produced in significant amounts by fermenting bacteria [18]. Acetate and/or propionate given intravenously or orally have been shown to reduce the serum concentration of free fatty acids $[19,20]$. It is reported that propionic acid infused into the cecum moderates the increase in plasma cholesterol caused by a casein diet [21]. Thus, SCFAs produced by cecal microflora must be related to lipid metabolism. The plasma total triglyceride level was higher in mice fed the guar gum-fructooligosaccharide diet than in those fed guar gum alone. It is reported that serum triglyceride levels are significantly lower in rats fed fructooligosaccharides [22] and oligofructose, which are chemically similar to fructooligosaccharides [18]. However, in our present study, the serum triglyceride concentration in mice fed guar gum was significantly lower than that in mice fed guar gum plus fructooligosaccharides. The difference in the plasma triglyceride concentration between the guar gum and guar gum plus fructooligosac- 
charides groups might be related to a difference in floral metabolism, floral composition, or both.

\section{REFERENCES}

1. Khan, A.R., Khan, G.Y., Mitchel, A., and Qadeer, M.A. (1981): Effect of guar gum on blood lipids. Am. J. Clin. Nutr., 34, 2446-2449.

2. Fernandez, M.L. (1995): Distinct mechanisms of plasma LDL lowering by dietary fiber in the guinea pig: Specific effects of pectin, guar gum, and psyllium. J. Lipid Res., 36, 23942404.

3. Garcia-Diez, F., Garcia-Mediavilla, V., Bayon, J.E., and Gonzalez-Gallego, J. (1996): Pectin feeding influences fecal bile acid excretion, hepatic bile acid and cholesterol synthesis and serum cholesterol in rats. J. Nutr., 126, 1766-1771.

4. Borel, P., Lairon, D., Senft, M., Chautan, M., and Lafont, H. (1989): Wheat bran and wheat germ: Effect on digestion and intestinal absorption of dietary lipids in the rat. Am. J. Clin. Nutr., 49, 1192-1202.

5. Kay, R.M. (1982): Dietary fiber. J. Lipid Res., 23, 221-242.

6. Kay, R.M., and Truswell, A.S. (1977): Effect of citrus pectin on blood lipids and fecal steroid excretion in man. Am. J. Clin. Nutr., 30, 171-175.

7. Hosoya, N., Dhorranintra, B., and Hidaka, H. (1988): Utilization of $\mathrm{U}^{-14} \mathrm{C}$ fructooligosaccharides in man as energy resources. J. Clin. Biochem. Nutr., 5, 67-74.

8. Stone-Dorshow, T., and Levitt, M.D. (1987): Gaseous response to ingestion of a poorly absorbed fructooligosaccharide sweetener. Am. J. Clin. Nutr., 46, 61-65.

9. Berggren, A.M., Björck, I.M.E., Nyman, M., and Eggum, B.O. (1993): Short-chain fatty acid content and $\mathrm{pH}$ in caecum of rats given various sources of carbohydrates. J. Sci. Food Agric., 63, 397-406.

10. Kohashi, M., Takahashi, A., and Iwai, K. (1990): Effect of a histidine-excess diet on a tetrahydrofolylpolyglutamate pattern in rat liver. J. Nutr. Sci. Vitaminol., 36, 11-19.

11. Hirayama, K., Itoh, K., Takahashi, E., and Mitsuoka, T. (1995): Comparison of composition of faecal microbiota and metabolism of faecal bacteria among 'human-flora-associated' mice inoculated with faeces from six different human donors. Microb. Ecol. Health Dis., 8, 199-211.

12. Allain, C.C., Poon, L.S., Chan, C.S.G., Richmond, W., and Fu, P.C. (1974): Enzymatic determination of total serum cholesterol. Clin. Chem., 20, 470-475.

13. Spayd, R.W., Bruschi, B., Burdick, B.A., Dappen, G.M., Eikenberry, J.N., Esders, T.W., Figueras, J., Goodhue, C.T., LaRossa, D.D., Nelson, R.W., Rand, R.N., and Wu, T.-W. (1978): Multilayer film elements for clinical analysis: Applications to representative chemical determinations. Clin. Chem., 24, 1343-1350.

14. Hidaka, H., Eida, T., Tarizawa, T., Tokunaga, T., and Tashiro, Y. (1986): Effects of fructo-oligosaccharides on intestinal flora and human health. Bifidobact. Microflora, 5, 3750.

15. Hirayama, K., Mishima, M., Kawamura, S., Itoh, K., Takahashi, E., and Mitsuoka, T. (1994): Effects of dietary supplements on the composition of fecal flora of human-floraassociated (HFA) mice Bifidobact. Microflora, 13, 1-7.

16. Weaver, G.A., Tangel, C.T., Krause, J.A., Alpern, H.D., Jenkins, P.L., Parfitt, M.M., and Stragand, J.J. (1996): Dietary guar gum alters colonic microbial fermentation in azoxymethane-treated rats. J. Nutr., 126, 1979-1991.

17. Campbell, J.M., Fahey, G.C., Jr., and Wolf, B.W. (1997): Selected indigestible oligosaccharides affect large bowel mass, cecal and fecal short-chain fatty acids, $\mathrm{pH}$ and microflora in rats. J. Nutr., 127, 130-136.

18. Fiordaliso, M., Kok, N., Desager, J.-P., Goethals, F., Deboyser, D., Roberfroid, M., and Delzenne, N. (1995): Dietary oligofructose lowers triglycerides, phospholipids and choles- 
terol in serum and very low density lipoproteins of rats. Lipids, 30, 163-167.

19. Crouse, J.R., Gerson, C.D., Oscarli, L.M., and Liebers, C.S. (1968): Role of acetate in the reduction of plasma free fatty acids produced by ethanol in man. J. Lipid Res., 9, 509-513.

20. Wolever, T.M.S., Spadafora, P., and Eshuis, H. (1991): Interaction between colonic acetate and propionate in humans. Am. J. Clin. Nutr., 53, 681-687.

21. Ebihara, K., Miyada, T., and Nakajima, A. (1993): Hypocholesterolemic effect of cecally infused propionic acid in rats fed a cholesterol-free, casein diet. Nutr. Res., 13, 209-217.

22. Tokunaga, T., Oku, T., and Hosoya, N. (1986): Influence of chronic intake of new sweetener fructooligosaccharide (Neosugar) on growth and gastrointestinal function of the rat. $J$. Nutr. Sci. Vitaminol., 32, 111-121. 